\title{
LA GESTIÓN DE LA CRISIS DE LOS CUIDADOS Y SU RELACIÓN CON LA FEMINIZACIÓN DE LAS MIGRACIONES. ANÁLISIS COMPARATIVO DE LOS MODELOS DE ESPAÑA Y CHILE
}

\author{
Managing the crisis in care and its relationship with the \\ feminisation of migrations. A comparative analysis of the \\ models in Spain and Chile
}

\author{
María Luisa SetiÉn SANTAMaría* \\ ElAine Acosta GONZÁLEZ**
}

\section{Resumen}

En los últimos años, España y Chile se han constituido en casos emblemáticos de países que han atravesado una profunda crisis del cuidado, y en respuesta a dicho fenómeno han comenzado a importar mano de obra inmigrante, fundamentalmente femenina, para hacerse cargo de las labores de reproducción social. La presente ponencia tiene por objetivo identificar y comparar los modelos de gestión de la crisis de los cuidados en España y Chile, conociendo cómo la política social ha dado respuesta

\footnotetext{
* María Luisa Setién Santamaría

Universidad de Deusto

Avenida de las Universidades, 24. 48007 Bilbao

Teléfono: 944139323 Fax: 944139378

mlsetien@ets.deusto.es

** Elaine Acosta González

Universidad de Deusto

Avenida de las Universidades, 24. 48007 Bilbao

Doctoranda del programa en Estudios Internacionales e Interculturales de la Univer-

sidad de Deusto

Profesora e investigadora de la Universidad Alberto Hurtado en Chile

Teléfono: 9444544 14/695 875126

elaineacosta72@gmail.com
} 
en materia de distribución de la responsabilidad social del cuidado de personas dependientes entre el Estado, el mercado, la comunidad y la familia y qué régimen de bienestar social se encuentra asociado a dicha distribución. Los resultados aquí presentados forman parte de una investigación en curso, cuya metodología aplicada ha consistido en análisis bibliográfico, análisis de la normativa y la política social y análisis estadístico de fuentes secundarias.

Palabras clave: crisis del cuidado, feminización de las migraciones, personas dependientes, organización social del cuidado.

\begin{abstract}
In recent years, Spain and Chile have become emblematic cases of countries that have gone through a major crisis in care, and which have responded by importing mainly female immigrants for this reproductive labour. This paper identifies and compares this crisis of care is managed in Spain and Chile, examining how social policy has responded in terms of spreading the responsibility for dependent care between the State, the market, the community and the family, and which social welfare regimen is linked to this spread. The results form part of an ongoing investigation, using an applied methodology involving bibliographic analysis, normative and social policy analysis, and statistical analysis of secondary sources.
\end{abstract}

Keywords: care crisis, feminisation of migrations, dependent persons, social organisation of care.

\title{
1. Introducción
}

En la actualidad, siguen siendo las mujeres quienes continúan asumiendo gran parte del trabajo de cuidado remunerado y no remunerado y, al mismo tiempo, son ellas las que crecientemente demandan servicios de cuidado, produciéndose una feminización de la dependencia y de su atención. Diversos procesos, como el envejecimiento de la población y el incremento de la participación laboral femenina, han contribuido a poner en jaque el modelo previo de organización social de los cuidados, sustentado en la división sexual del trabajo.

Como resultado, se ha producido la llamada «crisis de los cuidados» y ha sido la migración una de las estrategias de resolución de la crisis que han surgido como respuesta. Se le adjudica a esta crisis el haber influido significativamente en la feminización de los procesos migratorios contemporáneos, toda vez que se ha abierto un conjunto de oportunidades laborales para las mujeres migrantes tanto en el servicio doméstico como en el ámbito de los cuidados de personas dependientes (Perez Orozco, 2007). Lo cierto es que a nivel social, la contribución de las mujeres inmigrantes ha sido crucial en la resolución del déficit de cuidados.

España y Chile forman parte del grupo de países que han experimentado un cambio significativo en su situación en relación con los flujos migratorios, 
pasando de ser países tradicionalmente proveedores de migrantes a recibir inmigrantes. En ambos casos, una de las características distintivas de este cambio tiene relación con la feminización de los flujos migratorios hacia ambos países, hecho que está estrechamente conectado con la mayor oferta de empleos que tradicionalmente han realizado las mujeres. Esto último se explica, entre otros factores, por la menor disponibilidad de las mujeres autóctonas para ejercer las tareas de cuidado. Como resultado, tanto en España como en Chile se ha comenzado a importar, de manera significativa en los últimos años, mano de obra inmigrante, especialmente femenina, para hacerse cargo de las labores de reproducción social.

En este contexto vale la pena preguntarse, en cada uno de los países, cuál ha sido la respuesta en materia de política social para enfrentar la crisis de los cuidados y qué modelos de gestión se han aplicado. En otras palabras, cómo se está distribuyendo tanto en Chile como en España la responsabilidad social del cuidado entre el Estado, el mercado, la comunidad y la familia y qué régimen de bienestar social se encuentra asociado a dicha distribución. Tomando en consideración estos antecedentes, este artículo tiene como objetivos, en primer lugar, dar cuenta de la actual crisis del cuidado que atraviesan las sociedades española y chilena y la relación de este fenómeno con la feminización de las migraciones contemporáneas; en segundo lugar, analizar comparativamente los modelos de gestión de la crisis del cuidado en España y Chile.

\section{Los cambios sociodemográficos en España y Chile y su relación con la crisis del cuidado}

El creciente déficit de cuidado de personas dependientes -aquellas que, por razones ligadas a la falta o pérdida de capacidades físicas, psíquicas o intelectuales, tienen necesidad de asistencia y/o ayuda para la realización de actividades de la vida diaria (Tellechea, 2005)- se debe a varios factores ${ }^{1}$. Entre ellos cuenta la incorporación de las mujeres al mercado laboral (que disminuye su disponibilidad como cuidadoras del hogar), los cambios en la estructura de las familias (ha disminuido el tamaño familiar), así como el envejecimiento de la población (lo que implica una mayor necesidad de cuidado durante la vida). Todo esto obliga a las familias a buscar apoyo para la provisión de

1. Aunque usualmente el término dependencia suele asociarse a las personas mayores, en este artículo lo estamos usando en un sentido más amplio, al considerar todas aquellas personas que pueden requerir de atención y cuidados porque no pueden hacer por sí mismas las actividades de la vida cotidiana. De tal modo que a la ancianidad y a la infancia, agregamos aquellos que se encuentran en situación de discapacidad y minusvalía, enfermedad crónica. 
Tabla 1. Factores sociodemográficos que inciden en la crisis del cuidado

\begin{tabular}{|c|c|}
\hline $\begin{array}{l}\text { Factores relacionados con la } \\
\text { oferta de cuidado }\end{array}$ & $\begin{array}{c}\text { Factores relacionados con la } \\
\text { demanda de cuidado }\end{array}$ \\
\hline $\begin{array}{l}\text { Aumento de participación laboral } \\
\text { femenina } \\
\text { Cambios en estructura y dinámica } \\
\text { familiar (disminución del tamaño familiar, } \\
\text { privatización proyectos de vida). } \\
\text { Persistencia del modelo de hombre } \\
\text { proveedor-madre cuidadora }\end{array}$ & $\begin{array}{l}\text { Envejecimiento de la población } \\
\text { Aumento de la esperanza de vida } \\
\text { Elevación de los estándares de salud } \\
\text { pública }\end{array}$ \\
\hline
\end{tabular}

Fuente: Elaboración propia.

cuidado de sus integrantes más allá de lo que ellas mismas son capaces de proporcionar. La Tabla 1 resume, esquemáticamente, los aspectos más significativos que condicionan tanto la oferta como la demanda de cuidado de personas dependientes en ambos países.

En relación con la oferta de cuidado, la gran mayoría de los expertos coincide en que uno de los factores más importantes que estaría incidiendo en la crisis de los cuidados se debe al significativo cambio experimentado por las mujeres en su posición social actual a partir de su participación, cada vez más creciente, en el mercado laboral. En España comienza a registrarse a partir de los años noventa un importante incremento de la participación laboral femenina hasta sobrepasar en la actualidad el $40 \%$, mientras que la masculina se ha estabilizado. En Chile también aumentó la tasa de participación femenina sustancialmente en los últimos 20 años. Según datos de la Encuesta Nacional de Empleo, esta tasa de participación femenina era del 29,3\% en 1986 y del 40,9 \% en 2008.

Junto con el incremento de la participación laboral femenina, el modelo cultural de organización familiar predominante, que se sustenta en el rol de cuidadora de la mujer, está íntimamente relacionado con la «crisis de los cuidados». Las estadísticas muestran que la mayor parte de las mujeres españolas que trabajan lo hacen a tiempo completo y la participación de los hombres españoles en el trabajo doméstico es la menor de toda Europa (Eurostat, 1995 y Eurobarómetro, 1991, en Tobío y Díaz, 2003). En términos comparativos, Chile aparece como uno de los países que más enfatiza los costos en la vida familiar asociados al trabajo remunerado de la mujer, especialmente en el caso del cuidado de los hijos. ${ }^{2}$ Con lo antes dicho se confirma la hipótesis de que

2. Véase al respecto ver CEP. Mujer, trabajo y familia: Realidad, percepciones y desafíos. Análisis sobre la base de la encuesta CEP de diciembre 2002. Centro de Estudios Públicos. Puntos de Referencia 269, septiembre 2003. 
tanto en Chile como en España aún impera culturalmente el modelo de hombre proveedor-madre cuidadora, incluso cuando la mujer participa activamente en el mercado laboral. La presencia de nuevos modelos de división del trabajo doméstico es todavía incipiente en ambos países.

Adicionalmente, la familia ha estado sujeta a un conjunto de transformaciones que han alterado no sólo su estructura, sino también su dinámica de comportamiento. En primer lugar, hemos pasado de una familia extensa a un modelo de familia nuclear, que se ha replegado sobre sí misma y en la que la solidaridad intergeneracional no es una obligación prioritaria. En resumen, los procesos de privatización de los proyectos de vida individual han erosionado el control social para la asunción de responsabilidades familiares, generándose con ello discriminaciones y desigualdades que afectan fundamentalmente a las mujeres y personas dependientes.

En relación con los factores que explican el aumento de la demanda de cuidado aparece el envejecimiento de la población como uno de los más significativos. En España, en tan solo 20 años (1981-2002), el peso de la población mayor aumenta del 11,2\% al 17,1 \% (IMSERSO, 2005). Todavía más llamativo resulta el fenómeno conocido como el envejecimiento dentro del envejecimiento. En el mismo periodo ha crecido significativamente la población española más longeva, pasando a representar del 1,9\% al $4 \%$ de la población. En Chile, según datos del último Censo (2002), las personas mayores de 60 años constituyen el 11,4\% de la población nacional. Las estimaciones indican que de mantenerse las tendencias actuales, las proporciones de adultos mayores chilenos frente a menores de 15 años será de casi la mitad (Morales, sin año).

Junto con lo anterior, se ha registrado un incremento sin precedentes de la esperanza de vida en ambos países. En el caso de España, se ha pasado de los 40 escasos años de vida con que contaban las personas al nacer en las primeras décadas del siglo pasado, a los 83 años para las mujeres y a los 76 para los hombres en la actualidad (IMSERSO, 2005). Chile, junto a Costa Rica y Cuba, se sitúa entre los tres países con la esperanza de vida más alta de América Latina (INE, 2004). Los recién nacidos en Chile a principios del siglo XXI tienen una expectativa de vida en promedio de poco más de 77 años, casi el doble de la estimada en su momento para quienes nacieron a comienzos del siglo XX.

El incremento de la demanda de cuidado también está relacionado con el aumento de los estándares de salud pública. La elevación de la calidad de los tratamientos médicos para las personas discapacitadas y crónicamente enfermas, también alargan su esperanza de vida. En Chile, un 12,9 \% de la población padece algún tipo de discapacidad (FONADIS, 2004), mientras que en España, representa el 9 \% (Madariaga, 2008). Por último, a la demanda 
de estos grupos se suma la de la población menor de 18 años, que, si bien ha experimentado un proceso paulatino de disminución, continúa siendo responsabilidad prioritaria de las familias, especialmente en los sectores de bajos ingresos en el caso chileno (Acosta, Perticara y Ramos, 2007).

La incidencia e impacto tanto de los factores sociodemográficos como de los procesos de cambios sociales descritos anteriormente han erosionado el modelo previo de organización social de los cuidados, disminuyendo la oferta de cuidadores y aumentando la demanda de cuidado. Dicha demanda, al no poder ser satisfecha por la familia, los servicios sociales o el mercado local, está siendo crecientemente proporcionada por mujeres inmigrantes, tanto en España como en Chile.

\section{La importación de mano de obra inmigrante femenina como una respuesta a la crisis del cuidado en España y Chile}

Una de las características distintivas del aumento significativo de la participación femenina en los movimientos poblacionales está relacionada con la mayor oferta de empleos disponibles que tradicionalmente han realizado las mujeres. Al observar los flujos migratorios internacionales puede apreciarse un importante movimiento de las mujeres hacia España, como caso ilustrativo de los flujos migratorios sur-norte. En España, de cada 100 personas extranjeras residentes, esto es, con tarjeta o permiso de residencia en vigor, aproximadamente 45 son mujeres, lo cual indica que su presencia no es marginal. Este colectivo femenino ha aumentando en torno a un $162 \%$ entre 1997-20033.

Según datos del Observatorio Permanente de la Inmigración, desde 1997 los permisos de trabajo concedidos a mujeres en España aumentan anualmente un $10 \%$ y siete de cada diez permisos solicitados son para trabajar en el servicio doméstico. Por ejemplo, España es el destino preferente de las mujeres de países de Latinoamérica, especialmente por el idioma común ${ }^{4}$. En particular, se puede constatar la creciente feminización de algunas colonias de inmigrantes, particularmente provenientes de República Dominicana, Perú y Ecuador,

3. Es un crecimiento nada desdeñable, pero inferior al alcanzado en el mismo periodo por el grupo de los hombres inmigrantes (que han crecido un $200 \%$, desde 301261 a 904331), por lo que en el conjunto de España se observa un importante incremento de los flujos migratorios femeninos, aunque su peso relativo muestre una ligera tendencia descendente, rompiendo la línea evolutiva señalada a nivel mundial.

4. Es importante considerar que el mayor crecimiento de la población extranjera se ha producido en la población procedente de América -y más concretamente de Latinoamérica- que entre 2001 y 2007 ha aumentado su presencia en un $256 \%$, de modo que actualmente representa el 35 \% de la población extranjera residente en España. 
concentradas fundamentalmente en el sector de los servicios (Tobío y Díaz, 2003).

En los flujos migratorios sur-sur, Chile aparece como un país emergente de atracción de inmigrantes y los datos censales confirman la feminización cuantitativa de su inmigración. La inserción de la población activa inmigrante según ramas de actividad tiene como primeras mayorías al servicio doméstico (16\%) y al comercio minorista (13\%). El trabajo doméstico se ha convertido en la principal actividad económica desarrollada por mujeres inmigrantes, principalmente provenientes de Perús. De acuerdo con la información del Censo del 2002, el 71,5\% de las mujeres provenientes de ese país trabaja en esta actividad (Martínez, 2003). La población peruana resulta ser la que registra mayor concentración en el servicio doméstico (43\%).

Como resultado de la mencionada mayor presencia femenina en los movimientos poblacionales, no sólo se contribuye a resolver el déficit de cuidado en los lugares de destino, sino que adicionalmente se generan un conjunto de reordenamientos y transformaciones tanto en los hogares de origen. Para las mujeres migrantes se plantea tanto el problema del cuidado de su descendencia como de sus ascendientes, lo que se agrava dado el contexto de debilidad de los sistemas de protección social (Escrivá, 2005). La externalización del trabajo de cuidado, particularmente la realizada con mujeres inmigrantes, genera nuevos conflictos que se relacionan, por un lado, con la transformación de las relaciones sociales establecidas entre proveedores y receptores de cuidado, y por otro, con los sistemas que sostienen estos servicios, que generalmente reproducen estructuras de inequidad y discriminación, tanto para los trabajadores como para las personas necesitadas de cuidado. Amén de estas dificultades, lo cierto es que a nivel social, la contribución de las mujeres migrantes ha sido crucial en la resolución del déficit de cuidados.

\section{El rol del Estado y de las políticas sociales en la organización social del cuidado de personas dependientes}

El análisis sobre los regímenes de cuidado nos remite a ciertas conceptualizaciones del Estado en función del papel que éste ocupa en la distribución de dicha responsabilidad. Un régimen de cuidado refiere a las modalidades que adopta la distribución social del cuidado, o en otras palabras, a la organización

5. Dentro de los cuatro principales grupos migratorios hacia Chile (argentinos, peruanos, ecuatorianos y bolivianos) destaca Perú como el que más crecimiento ha experimentado en los últimos años, llegando a equipararse con la migración histórica hacia Chile liderada por los argentinos. 
que la provisión de cuidado tiene en un nivel macrosocial. Es de interés de este artículo conocer el rol que cumple el Estado en esta materia, ya sea adjudicándose la responsabilidad principal y directa de proveer servicios de cuidado $\mathrm{u}$ otorgando las medidas para que otros agentes -familia, comunidad o mercado- se hagan responsables y ejecutores del mismo.

Dependiendo del rol que juegue el Estado, los regímenes de cuidado, según Aguirre (2005), pueden clasificarse de dos formas: el régimen «familista» y el régimen «desfamilista». En el régimen familista, la responsabilidad principal corresponde a las familias y a las mujeres en las redes de parentesco, siendo la familia la unidad a quien se entregan los beneficios. Cuando ocurre que las mujeres trabajan además en forma remunerada, se desarrollan una serie de estrategias de conciliación entre trabajo y familia, estrategias que muchas veces tienden a reproducir, o hacen posible mantener, la división sexual del trabajo. ${ }^{6}$ Por su parte, en el modelo «desfamilista» se deriva la responsabilidad al Estado y al mercado, y es el individuo quien recibe los apoyos.

Los supuestos ideológicos que sustentan ambos regímenes de cuidado difieren. Mientras que el modelo familista supone la centralidad del matrimonio legal y la división sexual del trabajo, el desfamilista se basa en un cuestionamiento de la relación esfera pública/esfera privada y en políticas familiares activas. En resumen, el peso del Estado y la extensión de los servicios lucrativos, así como la participación de las familias y las redes informales de apoyo, están implicadas en las variaciones que se encuentran de estos regímenes.

En los últimos años, los países europeos han incrementado considerablemente las investigaciones comparativas sobre la génesis, fundamentos y situaciones de «crisis» de los sistemas de protección social. Dentro de lo que se ha denominado la «corriente dominante de investigación» sobre las políticas sociales, se destacan los aportes del sociólogo Gosta Esping-Andersen (1998) por cuanto han sido iluminadores al plantear un esquema sistemático para el análisis comparativo de los sistemas de protección social, facilitando la comprensión de las diferencias existentes entre los distintos Estados del Bienestar. Sin embargo, aún cuando ha sido muy útil la aportación del mencionado autor, desde la crítica feminista han surgido varias voces en desacuerdo con la escasa o nula consideración que la corriente dominante le ha concedido al papel que juega la familia en la estructuración de los sistemas de protección

6. Aguirre cita las estrategias propuestas por María Ángeles Durán, que consisten en: a) la reducción de objetivos tanto en el plano laboral como familiar, b) la delegación, que consiste en interrumpir la producción de un servicio para trasladarlo a otra persona y c) la secuencialización, que consiste en alternar la producción para la familia y para el mercado que es lo que buscan las excedencias y las licencias maternales. 
social $^{7}$. Por consiguiente, la tipología propuesta por Esping-Andersen ha sido ampliamente discutida y modificada ${ }^{8}$. En el debate, uno de los argumentos que a efectos de este artículo interesa destacar tiene relación con el concepto de familismo, que se propone sea definido con mayor detalle, desarrollando un dispositivo teórico sensible al género, que permita identificar las múltiples variaciones del familismo (Leitner, 2003).

$\mathrm{Al}$ aplicar la dimensión de género, pueden identificarse cuatro tipos ideales de políticas de acuerdo a la provisión del cuidado -opcionalmente familísticas, explícitamente familísticas, implícitamente familísticas y desfamilistas (Leitner, 2003)-, tal y como se muestra en la Tabla 2. El segundo eje en el que las feministas han trabajado está relacionado con el acceso a los derechos sociales de las mujeres y, en consecuencia, a la noción de ciudadanía social (Letablier, 2007). Siguiendo esta línea, Pautassi (2007) ha insistido en que la problemática del cuidado y quien lo ejerce remite necesariamente a un problema de ejercicio de derechos, reproducción de desigualdades y política pública en el que la evidencia empírica indica la perpetuación de situaciones de desigualdad en el tratamiento de una responsabilidad social ${ }^{9}$. Una de las expresiones concretas de este problema tiene que ver con los desafíos que las mujeres enfrentan para insertarse en igualdad de oportunidades en el mercado laboral y la manera en que se reparten los roles y la responsabilidad de las tareas de cuidado entre el Estado, la familia y el mercado de trabajo, quiénes asumen los costos y cómo son socialmente valoradas estas actividades. A continuación analizaremos cómo se produce esta distribución de responsabilidades de cuidado en España y Chile y qué modelos de gestión de la crisis se han implementado en cada caso.

\section{Los modelos de gestión de la crisis del cuidado de personas dependientes en España y Chile}

5.1. El modelo español de promoción de la autonomía personal y apoyo a la dependencia como un nuevo derecho social

El informe de resultados del estudio sobre Cuidado a la Dependencia e Inmigración (IMSERSO, 2005) concluye que el cuidado de personas dependientes que ejercen las mujeres en España descansa sobre un modelo familiar de reparto

7. Para más información sobre el desarrollo de esta crítica véase, Letablier, 2007.

8. El propio Esping-Andersen ha reconocido en su producción posterior la carencia de atención sistemática a la familia, como algo «dolorosamente evidente» (Esping-Andersen, 2000, citado en Añón y Miravet, 2005).

9. Letablier (2007) destaca cómo el enfoque comparativo ha sido muy útil para desvelar las distintas maneras de basar los derechos sociales y, en particular, su dimensión sexuada. 
Tabla 2. Tipos ideales de política de acuerdo a la provisión del cuidado aplicando un análisis de género

\begin{tabular}{|l|l|}
\hline \multicolumn{1}{|c|}{ Tipo de políticas } & \multicolumn{1}{c|}{ Descripción } \\
\hline Desfamiliarizadoras & $\begin{array}{l}\text { Fuerte desfamiliarización debido a la provisión de ser- } \\
\text { vicios de cuidado tanto del Estado como del mercado } \\
\text { y una débil familiarización. Los cuidadores familiares } \\
\text { están (parcialmente) descargados, pero el derecho fa- } \\
\text { miliar a cuidar no está valorado. }\end{array}$ \\
\hline $\begin{array}{l}\text { Implícitamente } \\
\text { familiarizadoras }\end{array}$ & $\begin{array}{l}\text { No ofrecen la desfamiliarización ni apoyan activamen- } \\
\text { te la función de cuidado en la familia a través de nin- } \\
\text { gún tipo de política familiarizadora. Sin embargo, la } \\
\text { familia sigue siendo el cuidador principal, dado que no } \\
\text { se ofrecen alternativas de cuidado. Es un tipo de polí- } \\
\text { tica que confía o depende implícitamente en la familia } \\
\text { cuando se trata de temas de cuidado. }\end{array}$ \\
\hline $\begin{array}{l}\text { Explícitamente } \\
\text { familiarizadoras }\end{array}$ & $\begin{array}{l}\text { Fortalecen a la familia en la función de cuidado a tra- } \\
\text { vés de políticas familiarizadoras. A través de ellas no } \\
\text { se ofrecen alternativas al cuidado familiar. Explícita- } \\
\text { mente se fortalece la función de cuidado en la familia } \\
\text { debido tanto a la carencia en la esfera pública y en el } \\
\text { mercado de provisión de cuidado como a la fuerte fa- } \\
\text { miliarización. }\end{array}$ \\
\hline $\begin{array}{l}\text { Opcionalmente } \\
\text { familiarizadoras }\end{array}$ & $\begin{array}{l}\text { Se fortalece el cuidado familiar pero, al mismo tiempo, } \\
\text { se ofrece la opción de ser (parcialmente) liberado de } \\
\text { las responsabilidades de cuidado. El «derecho» de la } \\
\text { familia a cuidar no es equiparado con la «obligación» } \\
\text { de la familia a cuidar. }\end{array}$ \\
\hline
\end{tabular}

Fuente: Elaboración propia en base a Leitner (2003).

desigual de roles, basado en el trabajo doméstico de la mujer y en su situación de inactividad económica. En España, al igual que en otros países del sur de Europa, la intervención del Estado para favorecer la compatibilidad entre la vida familiar y laboral aún es limitada, a pesar de los significativos avances que tanto en materia de normativa como de política social se han realizado en los últimos años. Estos avances han permitido colocar como un tema prioritario dentro de la política social actual y de los próximos años la atención a las necesidades de apoyos generalizados y la promoción de autonomía personal, reconociendo con el rango de derechos lo que antes eran ayudas dispersas y subsidiarias del cuidado familiar.

\subsubsection{Predominio del cuidado informal de personas dependientes}

En España, la atención y cuidados a las personas dependientes son prestados en más de un $70 \%$ de las situaciones mediante los denominados «cuidados 
Tabla 3. Cuidados en la familia de mayores dependientes, España, 2004

\begin{tabular}{|l|c|}
\hline & 2004 \\
\hline Edad cuidador & 53 años \\
\hline Hijas & $47 \%$ \\
\hline Problemas profesionales y económicos & $62 \%$ \\
\hline No pueden trabajar fuera de casa & $26 \%$ \\
\hline Ha dejado de trabajar & $11.7 \%$ \\
\hline Problemas con su pareja & $7 \%$ \\
\hline Cuidador y mayor viven juntos & $70 \%$ \\
\hline
\end{tabular}

Fuente: Torres, 2006.

informales» (Iglesias, 2006). La asistencia al dependiente está asegurada fundamentalmente por la familia, siendo en particular las mujeres (esposas e hijas, y en menor medida las nietas) las que asumen esta labor. Son los mayores dependientes, según IMSERSO, quienes de forma más marcada reciben los cuidados de parte de la familia, llegando a un $86,5 \%$.

Como se puede apreciar en la Tabla 3, el perfil social de la cuidadora que constituye, a decir de María Ángeles Durán (2006), el soporte básico del Estado de Bienestar español, es el de una mujer entre 40 y 65 años de edad, que no realiza tareas remuneradas fuera del hogar, o bien las lleva a cabo a tiempo parcial. Por lo general, dedica más de 40 horas semanales al cuidado del dependiente y asume casi en exclusiva dicha responsabilidad. Presenta, en la mayoría de los casos, dificultades económicas y le resulta difícil mantener sus relaciones sociales. Como resultado del trabajo de cuidado padece patologías múltiples, especialmente cansancio, trastornos del sueño y frecuentemente depresión.

Las necesidades de atención que en particular tienen las personas mayores dependientes implican una dedicación horaria de atención que no siempre puede ser asumida en exclusividad por la familia. A ello se agrega la complejidad de los requerimientos de cuidados que en muchas ocasiones necesitan de una formación previa o de supervisión profesional, que pocas veces se produce. En resumen, los estudios realizados han conseguido demostrar que el recurso a la ayuda informal para el cuidado de personas mayores dependientes aparece como una opción preferente, relacionada con la práctica de la autonomía residencial y el familismo típico de la sociedad española.

\subsubsection{Insuficiente cobertura de servicios de cuidado}

Los cambios producidos en la estructura social española a partir de la participación masiva de la mujeren el mercado laboral han puesto en evidencia la 
falta de estructuras de atención alternativas a los cuidados informales. Estudios recientes indican que los cuidados «formales» ${ }^{10}$-integrados por el conjunto de servicios provistos por entidades y organizaciones, tanto públicas como privadas, con y sin ánimo de lucro, y por personas que trabajan por su cuenta de manera remunerada- atienden aproximadamente a un porcentaje en torno al $27 \%$ de las necesidades (Iglesias, 2006).

De acuerdo al Libro Blanco sobre la atención a personas en situación de dependencia (IMSERSO), la oferta de servicios de cuidado es manifiestamente insuficiente, tanto para las necesidades actuales como para las que previsiblemente se demanden en el futuro. Los servicios sociales formales de atención prestados a las personas que precisan cuidados y viven en su domicilio tienen muy poca significación. El peso de los mismos se calcula en un $4,7 \%$ del total de cuidados recibidos (IMSERSO, 2005). Adicionalmente la colaboración entre los servicios formales y los cuidadores informales es muy escasa.

En el mismo estudio se consigna la escasa implantación de servicios sociosanitarios formalizados de proximidad (servicios de ayuda a domicilio, teleasistencia y centros de día). Frente a esta carencia, la llegada de mujeres inmigrantes con posibilidades de realizar tareas de cuidado a mayores dependientes y la posibilidad de residir en el propio domicilio del mayor, ha sido una estrategia crecientemente utilizada por las familias españolas. Sin embargo, esta salida a la crisis podría estar ocultando la limitación de los servicios de proximidad. Adicionalmente, las mujeres inmigrantes estarían llenando otro vacío o debilidad asociado a los servicios formales de cuidado. Se trata de las aptitudes psicosociales y de la ética del sacrificio con la que contarían estas mujeres, procedentes por lo general de una cultura tradicional de respeto y atención a las personas mayores.

A los problemas de cobertura habría que agregar los desajustes económicos generadas como consecuencia de la atención constante de una persona en situación de dependencia. Frente a esta realidad, el Estado español presenta uno de los índices más bajos en materia de gastos de protección social a la vejez, al compararlo con el conjunto de la comunidad europea. «En 2005, la media europea de gasto para los mayores fue de 2.929 euros por persona, cantidad muy superior a los 1.648 euros que se gastaron en España.» (Setién, 2009: 332)

10. La oferta formal de cuidados se integra fundamentalmente por los servicios de ayuda a domicilio, teleasistencia, plazas de estancia diurna y centros residenciales, de provisión pública y privada. 
5.1.3. Avances en materia de normativa y política social: la creación de un Sistema Nacional de Dependencia

En respuesta a estos problemas, en los últimos años ha podido constatarse una creciente preocupación en España por los fenómenos sociodemográficos asociados a la crisis del cuidado. Dicha preocupación se ha expresado en el debate público sobre la conciliación de la vida laboral y familiar, que derivó en la Ley 39/1999 para promover la conciliación de la vida familiar y laboral de las personas trabajadoras, así como en la discusión sobre la protección social de la situación de dependencia.

Como resultado se aprobó en el 2006 la Ley 39/2006 de Promoción de la Autonomía Personal y Atención a las personas en situación de dependencia (conocida como Ley de Dependencia), que actúa como principal norma reguladora de la atención a las personas en tal condición en España. Entre sus objetivos se encuentra fomentar la promoción de la autonomía, evitando al máximo la institucionalización de las personas mayores dependientes, así como la optimización de los recursos públicos y privados en la prestación de servicios de cuidado. Con la aprobación de esta ley se ha configurado un derecho subjetivo fundamentado en los principios de universalidad, equidad y accesibilidad, desarrollando un modelo de atención integral al ciudadano. Para hacer realidad su mandato se ha diseñado un Sistema para la Autonomía y Atención a la Dependencia (SAAD), constituido en el organismo encargado de canalizar y optimizar los recursos públicos y privados. Los servicios que comprende dicho sistema van desde la prevención, la teleasistencia y ayuda a domicilio hasta las prestaciones económicas que pueden obtener las personas en situación de dependencia. Dada su reciente creación, quedan pendientes de evaluación los alcances, limitaciones o vacíos que pueda estar teniendo su aplicación práctica.

\subsection{El modelo chileno de máxima responsabilidad privada}

En el modelo chileno se produce una dependencia sistemática de la familia para la provisión de trabajo de cuidado y servicios, basada en los principios de subsidiaridad. A pesar de la escasez de estudios, las estadísticas disponibles permiten extender esta afirmación al resto de los grupos de personas dependientes.

\subsubsection{Predominio del cuidado informal de personas dependientes}

En Chile existe un fuerte predominio del cuidado informal que atraviesa los distintos grupos de personas dependientes. Este predominio se refleja, en 
primer lugar, en las características de la oferta y la demanda actual de cuidado infantil, por citar el grupo más estudiado. ${ }^{11}$ En el caso de los menores, alrededor del $80 \%$ del cuidado es proporcionado por las madres o familiares. (Acosta, Perticara y Ramos, 2007). Para el caso de las personas discapacitadas, se estima que hasta en un $88 \%$ del total de cuidados prestados corresponde a cuidados de tipo informal (Herrera, 2007) y el $68,71 \%$ de los apoyos son proporcionados por un familiar (FONADIS-INE, 2004), como puede apreciarse en la Tabla 4.

En el caso de los adultos mayores, el perfil de los cuidadores de adultos mayores corresponde en más de un $80 \%$ a los familiares, en particular a las mujeres que representan entre el 75 y el $84 \%$ de quienes proveen de cuidados a ancianos (Zavala y otros, 2001). Lejos de la creencia común de que el anciano en la sociedad contemporánea ha sido abandonado por su familia, la evidencia empírica en Chile muestra una realidad muy distinta. La familia sigue siendo el principal sostén para los adultos de edad avanzada (Guzmán y Huenchuan, 2005).

\subsubsection{Escasez de servicios de cuidado coherentes con la realidad de participación laboral de la mujer}

El mencionado predominio del cuidado informal está estrechamente relacionado con las características de la oferta de servicios de cuidado para los distintos grupos de personas dependientes en Chile. En general, se trata de una oferta insuficiente en términos de cobertura y poco flexible y ajustada a la realidad de participación creciente de la mujer en el mercado laboral. Los estudios han constatado, entre otros problemas, poca disponibilidad de lugares de cuidado cercanos al domicilio, dificultades con el traslado, alto costo económico y riesgos para la salud -particularmente en el caso de los menores- como algunos de los problemas relacionados con la oferta de servicios de cuidado (Acosta, Perticara y Ramos, 2007).

El alto costo de la atención requerida por las personas dependientes, en particular, los discapacitados y ancianos enfermos, y la dificultad de los servicios asistenciales para atender las múltiples demandas de estos grupos, provoca que se traspase a los cuidadores familiares e informales prácticamente la totalidad de la responsabilidad y costo del cuidado. Éstos, no disponen muchas

11. Al respecto pueden consultarse los estudios «Oferta Laboral Femenina y Cuidado infantil» y «Modelos de Cuidado Infantil y Participación Laboral Femenina. Estrategias de conciliación entre el rol laboral y de cuidado en mujeres pertenecientes a sectores de bajos ingresos». 
Tabla 4. Discapacidad según quienes prestan apoyo.

Frecuencia y distribución porcentual, Chile 2004

\begin{tabular}{|l|r|c|}
\hline & N & $\%$ \\
\hline Amigos & 325 & 0,002 \\
\hline Cuidadora & 4978 & 0,24 \\
\hline Familiares & 1421066 & 68,71 \\
\hline Vecinos & 6282 & 0,30 \\
\hline Nadie & 635421 & 30,73 \\
\hline Total & 2068072 & 100 \\
\hline
\end{tabular}

Fuente: FONADIS, 2004.

veces del tiempo ni las competencias necesarias para brindar un cuidado de calidad. «Los cuidados de salud que se dejan de hacer en el sistema institucional público o privado son realizados en el espacio doméstico, la mayor parte por mujeres, que deben absorber los costos de la salud ahorrados por seguros públicos y privados sin ninguna visibilidad ni retribución.» (Observatorio de Equidad de Género en Salud-Chile, 2004)

Por su parte, en relación con los apoyos sociales disponibles para las personas con discapacidad, el primer estudio nacional sobre la discapacidad en Chile arroja algunos datos interesantes. El 70 \% de las personas con discapacidad cuenta con algún nivel de apoyo de una tercera persona, mientras que el $30 \%$ restante no tiene o no requiere de terceras personas que les preste apoyo (FONADIS, 2004). El $68 \%$ de las personas con discapacidad recibe apoyo de sus familiares, mientras que el resto de personas no es significativo en términos de porcentajes (amigos, cuidadores, vecinos).

5.2.3. Política social y normativa para el cuidado de personas dependientes: una tarea pendiente

Frente a la situación anterior, es evidente la necesidad de que la política social en Chile debe ajustarse a las nuevas necesidades y demandas de protección social y cuidado. En respuesta a ello se han producido avances tanto en el ámbito normativo como en el de la política, pero de manera diferenciada por grupos de personas dependientes. En el área de la infancia se han dado los pasos más significativos, creándose el Sistema de Protección Integral a la Infancia (Chile Crece Contigo) ${ }^{12}$. Se trata de un sistema que tiene como misión

12. Chile Crece Contigo forma parte además del recientemente creado Sistema de Protección Social comprometido por el Gobierno chileno hasta este año 2010. 
acompañar, proteger y apoyar integralmente, a todos los niños y sus familias, a través de acciones y servicios de carácter universal. Al mismo tiempo, focalizar apoyos especiales a aquellos que presenten alguna vulnerabilidad mayor. Quedan por evaluar, sin embargo, los avances registrados a partir de la implementación de esta política. Por el momento, sabemos que el gasto público en cuidado de menores y en la educación preescolar en Chile es de alrededor del $0,1 \%$ del PIB en 2007, cifra baja comparada con el promedio del 0,25\% que destina la OCDE.

Por su parte, la intervención estatal frente al envejecimiento está delineada en una política nacional promulgada en 1996. En ella, el Estado asume un rol subsidiario a favor del adulto mayor, tratando de superar el enfoque predominantemente asistencial que había existido hasta entonces. De esta forma, el Estado delimita responsabilidades frente al cuidado del adulto mayor, propendiendo a un papel activo por parte de la familia en primera instancia y también de la comunidad. Se pone un gran énfasis en que la responsabilidad por el adulto mayor se realice sin separarlo de su entorno habitual, promoviendo su integración activa al medio social.

Es en relación con la Seguridad Social, el campo donde con más fuerza se expresa el predominio o presencia de la economía de mercado, a través de cómo se compone el sistema de pensiones. Por tales razones, dentro de la nueva política pública en materia de protección social también se ha considerado la Reforma Previsional (Ley 20.255), que viene a cumplir la mayor promesa del gobierno de la presidenta Bachelet, al crear un nuevo sistema de pensiones que protege y cuida a todos los chilenos, desde la cuna hasta la vejez. Por su reciente creación todavía es muy pronto para poder realizar una evaluación de su impacto. Lo que sí podemos anticipar es que por sí sola esta reforma previsional no resolverá los múltiples problemas relacionados con el envejecimiento de la población y la carga de cuidado que ello representa para las familias chilenas.

\section{Reflexiones finales: hacia una política democrática del cuidado}

Al comparar los casos de España y Chile, se ha reafirmado la estrecha conexión existente entre la actual crisis del cuidado y el proceso de feminización de las migraciones. La importación de mano de obra femenina para realizar las labores de reproducción social es una estrategia crecientemente utilizada por las familias, tanto en los flujos migratorios sur-norte como en los sur-sur, siendo España y Chile casos ilustrativos de este fenómeno. Sin embargo, a nivel de política social la respuesta a la crisis de los cuidados ha tenido énfasis 
e impactos diferentes en materia de distribución social de la responsabilidad del cuidado en los dos países estudiados.

En cuanto a los modelos de gestión de la crisis del cuidado, los países estudiados comparten un modelo familista en tanto estructura normativa que modela la responsabilidad social del cuidado. Esta estructura confiere a la familia un rol central en la procura del bienestar, asociado a las asunciones ideológicas y parámetros de tipo simbólico, cultural y normativo, mencionados con anterioridad. Sin embargo, y siguiendo los aportes de Leitner, difieren en materia de política social, por cuanto en el caso español predomina una política de familiarismo implícito, mientras que el caso chileno se puede clasificar como de familiarismo explícito.

En el caso español, el Estado ha empezado a asumir crecientemente parte de las competencias cuidadoras que tradicionalmente tenían las familias y, en consecuencia, tanto el sistema de salud como los servicios sociales han liberado tiempo familiar de cuidado a las personas. En el caso chileno, en cambio, continúa predominando un modelo de máxima responsabilidad privada en relación con el cuidado de personas dependientes, siendo la familia, en particular las mujeres, la que asume la mayor parte de la carga de cuidados, sin que ésta sea debidamente reconocida a nivel público. A pesar de los recientes esfuerzos del actual gobierno en la construcción de un Sistema de Protección Social, existen importantes vacíos a nivel normativo y de la política social que permiten garantizar adecuadamente los derechos y deberes de las personas que requieren cuidados, así como de sus cuidadores.

De los casos estudiados se desprenden algunas lecciones. En primer lugar, la necesidad de estimular y consolidar el debate y reconocimiento público del cuidado de personas dependientes como un problema público, en el que el Estado tiene una gran responsabilidad. En segundo lugar, tomando en consideración los aportes de los estudios comparados sobre regímenes de protección social, se deriva la necesidad de continuar con el estudio del comportamiento de esta problemática y su relación con la feminización de las migraciones. Para ello, será imprescindible incorporar en el análisis a otros flujos migratorios y los marcos normativos que definan y garanticen los derechos y deberes de las personas que requieren cuidados, así como de sus cuidadores, en particular de las mujeres inmigrantes. 


\section{Bibliografia}

Acosta, E., M. Perticara y C. Ramos, «Oferta Laboral Femenina y Cuidado Infantil», en Desafíos al Desarrollo de Chile: Elementos para el Diálogo de Políticas, BID, Santiago de Chile, 2007.

AguirRe, R., Los cuidados familiares como problema público y objeto de políticas, reunión de expertos Políticas hacia las familias, protección e inclusión social, CEPAL, Montevideo, 2005.

AÑón, M. J. y P. Miravet, «Paradojas del familiarismo en el Estado del Bienestar: mujeres y renta básica», en Cuadernos de Relaciones Laborales, 23, n. ${ }^{\circ}$ 2, 2005, pp. 101-121.

DurÁn, M. ${ }^{a}$ Ángeles, «Dependientes y cuidadores: el desafío de los próximos años», Revista del Ministerio de Trabajo y Asuntos Sociales, n. ${ }^{\circ}$ 60, Madrid, 2006.

ESCRIVÁ, M. a Ángeles, «Peruanos en España. ¿De migrantes a ciudadanos?», en El Quinto suyo: Transnacionalidad y Formaciones Diaspóricas en la Migración Peruana, Lima, Instituto de Estudios Peruanos, 2005.

ESPING ANDERSEN, Gosta, The three worlds of welfare capitalism, New Jersey, Princeton University Press, 1998.

FONADIS (Fondo Nacional de la Discapacidad), Resultados. Primer estudio nacional de la discapacidad en Chile, Santiago de Chile, 2004.

- Apartado Género. Primer estudio nacional de la discapacidad en Chile, Santiago de Chile, 2004.

Guzmán, J. M. y S. Huenchuan, Políticas hacia las familias con adultos mayores: el desafío del derecho al cuidado en edad avanzada, Santiago de Chile, CEPALCELADE, 2005.

HERRERA, Eduardo, Cuidadoras y cuidadores de personas dependientes y enfoque de género en Chile, Santiago de Chile, FONADIS, 2007.

IGLESIAS, Pilar, "Sistema Nacional de Dependencia», en Revista del Ministerio de Trabajo y Asuntos Sociales, n. ${ }^{\circ}$ 60, Madrid, 2006.

IMSERSO, Cuidados en la vejez. El apoyo informal, Madrid, 1995.

- Cuidado a la dependencia e inmigración. Informe de Resultados, Colección de Estudios, Serie Dependencia, Madrid, 2005.

LEITNER, Sigrid, «Varieties of familialism», en European Societies, 5 (4), 2003, pp. 353-375.

LETABLIER, M. T., «El trabajo de "cuidados" y su conceptualización en Europa», en Trabajo, Género y tiempo social, C. Prieto, (ed.), Madrid, Editorial Complutense, 2007.

MADARIAGA ORTUZAR, Aurora, «Los servicios de ocio de las asociaciones de discapacidad», tesis doctoral, Bilbao, Universidad de Deusto, 2008. 
MARTínEZ, Jorge, El encanto de los datos. Sociodemografía de la inmigración en Chile según el Censo de 2002, Santiago de Chile, CEPAL, 2003.

Morales, María E., Chile envejece: Prospectiva de los impactos políticos y sociales de este fenómeno hacia el bicentenario, [en línea], <http://www.gerontologia. uchile.cl/docs/chien.htm>.

PAUTASSI, Laura, «El cuidado como cuestión social desde un enfoque de derechos», Unidad Mujer y Desarrollo, Santiago de Chile, CEPAL, 2007.

PÉrez Orozco, Amaia, «Cadenas globales de cuidado», serie Género, migración y desarrollo, documento de trabajo, 2, Naciones Unidas, INSTRAW, 2007.

SETIÉN, María Luisa, "Género y cuidados a las personas en el espacio doméstico», en Inguruak, Revista Vasca de Sociología y Ciencia Política, n. ${ }^{\circ} 22$, diciembre, 1998.

— «Gasto social en España», en Políticas Sociales, Carmen Alemán Bracho, (coord.), Pamplona, Thomson Reuters, Civitas, 2009, pp. 309-340.

TEllecheA, Lourdes, Cuidados permanentes de las personas mayores, documento de trabajo, Buenos Aires, [en línea], <www.eclac.cl>.

TOBíO, Constanza y Magdalena DíAz, Las mujeres inmigrantes y la conciliación de la vida familiar y profesional, Comunidad de Madrid, Dirección General de la Mujer, Consejería de trabajo, 2003.

TORRES, Julio Alberto, «Libro Blanco para las personas en situación de dependencia en España», en Revista del Ministerio de Trabajo y Asuntos Sociales, n. ${ }^{\circ}$ 60, Madrid, 2006.

VICENTE, Trinidad y María Luisa SETIÉN, «Modelos migratorios femeninos», en Diversidad migratoria. Distintos protagonistas, diferentes contextos, Julia González y María Luisa Setién (eds.), Bilbao, Universidad de Deusto, 2005.

Zavala y otros, Características sociodemográficas de los cuidadores de ancianos, ponencia presentada al IV Congreso Chileno de Antropología, Universidad de Chile, 2001, [en línea], <www.uchile.cl>.

\section{Nota:}

Este artículo forma parte del Proyecto FEM2009-09007 (Subprograma FE$\mathrm{ME}$ ): «Crisis del cuidado y migración. Análisis comparativo de flujos migratorios feminizados: sur-norte y sur-sur», financiado por el Ministerio de Ciencia e Innovación, España, 2009-2012. 